\title{
Information and Observability - Some Notes on the Economics of Moral Hazard and Insurance *
}

\author{
by Roland Eisen **
}

\section{Introduction}

Moral hazard refers to the tendency that insurance protection itself alters an individual's incentives to prevent loss or to take specific actions (as e.g. care), and changes, therefore, the probability and the magnitude of losses underlying the calculations of insurance companies. The source of this incentive problem is the asymmetric information of agents resulting from the difficulty to discriminate between actions of insured on the one hand, and exogenous uncertainty on the other hand: The insurer cannot completely define his risk, in most cases he only observes an outcome which is a mixture of risk and action (Arrow [1970], p. 142).

Owing to the incomplete observability of the insured's behaviour, and, therefore, the imperfect enforceability of a specific (desirable) behaviour, it is in general impossible to reach a Pareto-optimal risk allocation. Also, such an optimal risk spreading does not induce the right incentives for the choice of suitable actions. Therefore, only a second-best solution is possible representing an 'optimal' compromise between the two " conflicting goals of furthering risk spread and providing appropriate incentives" (Spence and Zeckhauser [1971], p. 385).

That second-best solution results in incomplete coverage against loss, subjecting the insured to some financial risk. To what extent this partial coverage (co-insurance) induces an incentive to take specific measures (e.g. care) depends on the costs of preventing losses $\mathbf{1}$.

* This is a thoroughly revised version of a paper delivered at the Seventh Seminar of the European Group of Risk and Insurance Economists, Nottingham, September 10-12, 1980. For valuable discussions I had to thank the participants, and especially Dr. M. Dietsch. Naturally, the usual caveat applies.

** University of Munich.

1 This question was recently studied by Shavell [1979] who finds that "the optimal insurance policy under moral hazard ... offers partial rather than full coverage if the cost of taking care is sufficiently low, but the level of coverage approaches full coverage as the cost of taking care tends to zero" (p. 546). 
An obvious remedy to this difficulty is to incur expenses to monitor or control the actions of the insured and to use this information in the insurance contract. Monitoring of the behaviour gives an actor an incentive to prevent loss either by allowing the insurer to raise the insurance premium or to reduce the amount of coverage paid in the event of a loss in accordance with the observed behaviour.

In some simple cases perfect monitoring may be possible and a first-best solution attainable with full coverage and optimal risk sharing. In general, however, perfect monitoring of the insured's action is impossible or prohibitively expensive. Optimal or efficient contracts depend then on the "technology of observation", indicating what is observable and to what costs.

In the following two different models leading to very distinct results are discussed. The comparison of these two models reveals intuitively the value of additional information. It is then asked whether partial or incomplete monitoring with partial and incomplete information as to the behaviour of the insured is worthwhile or valuable.

\section{Optimal contracts when the insurer has different information on action and outcome - Some previous results}

Spence and Zeckhauser [1971] classify insurance policies "according to their structural characteristics in three areas. The first is (1) the presence or absence of individual choice ... Where there is room for individual choice, we will be concerned with (2) the sequencing of moves between the individual and nature, and (3) the information state... monitored by the insurer. This information state... will be a function of the act of nature and the action of the individual" (p. 380). Here we choose two interesting, but very different structures.

The first model (see Eisen [1979], section 5.3) describes the case that the insured chooses an activity (or activity level) $a$ after Nature has "chosen" and state of nature $s(s=1,2, \ldots, S)$ has occurred (" exogenous uncertainty"). The insurer can only monitor $a$ (the activity, e.g. care), but not $s$ (the state). Here the variable $a$ can represent a certain level of investment (to reduce the magnitude of loss or to reduce the probability of loss, as e.g. sprinklers or anti-theft devices) or the purchase of certain goods or services (e.g. medical services). Subject to the simplifying condition that the company maximizes the utility of its members the problem is to choose optimal insurance policies.

An optimal insurance policy is described by a payment function which operates as an "optimal incentive scheme", i.e. as a solution to the above mentioned tradeoff between risk spreading and incentives. Here the payment function combines the premium (as a function of the coverage) and the coverage. It is assumed that given the coverage, there is a unique premium such that the insurer breaks even : the sum of all premium incomes is equal to the sum of all losses paid, the so-called collective principle of equivalence is valid. 
The result of this maximization problem is : If the insurer can only monitor the activity level $a$, the insured does not pay the full marginal costs under the optimal insurance policy under moral hazard, hence too much insurance (coverage) will be purchased. Dependent on the underlying probability distribution it is shown that the "optimal contract or payment function" is in general not linear, deductibles and co-insurance rates are then only approximations to the "optimum". More appropriate results may be obtained through sophisticated bonus-malus systems.

In this model, however, two unmentioned problems are hidden : First, it is assumed that an optimum exists, but expected utility may not be concave in the activity $a$, and there does not seem to be any simple condition on the probability function that would garantee concavity. Second, the optimal solution must be differentiable. But Mirrlees [1974] has given an example that shows that in general there is no (unique) optimum.

These difficulties are dealt with in the second model (see Eisen [1976]). Here some assumptions are altered: The insured has to choose his action $a$ before the state $s$ is known, the insurer cannot monitor the activity, he observes only the outcome, the mixture of risk and action ${ }^{2}$.

Following Mirrlees [1974] the explicit consideration of the state $s$ is eliminated, and $x$ (the loss) is viewed as a random variable with the conditional distribution of $x$ with respect to $a$, the insured's action (see also Eisen [1976]). This distribution function is $F(x, a)$ given $a$. Given a distribution of $s, F(x, a)$ is simply the distribution induced on $x$ via $x=x(a, s)$. Therefore, $s=F(x, a)$, and hence $x=x(a, F(x, a))$. It is easy to see that $x_{a} \leqslant 0$ implies $F_{a}(x, a) \leqslant 0$. In order that a change in $a$ has a nontrivial effect on the distribution of $x$, it is assumed that for every $a F_{a}(x, a)<0$ for some $x$-values. The density function $f(x, a)=F_{x}$ is then given by $f(x, a)=1 / x_{s}(a, F(x, a))$, and it is assumed that $f_{a}$ and $f_{a a}$ are well-defined for all $(x, a)$.

Let $\Phi(x)$ be the payment function, the essential characteristic of the insurance contract (or policy), combining premium paid and indemnity received; then the insurer

$$
\underset{\Phi(x)}{\operatorname{maximizes}} \int U(\Phi(x), a) f(x, a) d x
$$

such that

$$
\int[x-\Phi(x)] f(x, a) d x=0,
$$

i.e. the insurance contracts break even, implying that the insurer is risk neutral (owing to the fact that an insurer's risk is borne by many stockholders, and that the risks insured are many, small and approximately independent); and the insured

$$
\underset{a}{\operatorname{maximizes}} \int U(\Phi(x), a) f(x, a) d x \text {. }
$$

2 In Eisen [1976], it was erroneously written that the insurer can monitor the activity. I thank F. Breyer (University of Heidelberg) for pointing out this mistake. 
The utility function, $U$, of the insured ${ }^{3}$, whose first argument represents the insured's netto compensation or wealth and whose second argument is the action taken by the agent, is continuously differentiable, strictly concave in wealth (i.e. the insured is risk averse), and it is assumed that $\partial U / \partial a<0$. As shown in Eisen [1976] — with slightly different notation - this yields the following first-order condition for the choice of $\Phi(x)$ (for all $x)$ :

$$
\begin{aligned}
& U_{w}(\Phi(x), a) f(x, a)+\lambda U_{w a} f(x, a) \\
& +\lambda U_{w}(\Phi(x), a) f_{a}(x, a)-\mu f(x, a)=0 .
\end{aligned}
$$

with $\lambda$ and $\mu$ as Lagrange multipliers of the constraints (3) and (2) respectively.

Hence

$$
\frac{\lambda U_{w a}-\mu}{U_{w}}=-\left[1+\lambda \frac{f_{a}(x, a)}{f(x, a)}\right] .
$$

The action $a$ is given by

$$
\int U_{a}(\Phi(x), a) f(x, a) d x+\int U(\Phi(x), a) f_{a}(x, a) d x=0
$$

and $\lambda$ is given as the solution of

$$
\begin{aligned}
& \lambda\left\{\int U_{a a}(\Phi(x), a) f(x, a) d x+2 \int U_{a}(\Phi(x), a) f(x, a) d x\right. \\
& \left.+\int U(\Phi(x), a) f_{a a}(x, a) d x\right\}+\mu \int(x-\Phi(x)) f_{a}(x, a) d x=0 .
\end{aligned}
$$

The first-best or Pareto optimal risk spreading is achieved if the marginal utility of wealth of the insured is constant (or the same over all states of nature, if statedependent utility functions and social risks are excluded). But, one of the most interesting results of this model is that there is no first-best solution (called ' control optimum') achievable through insurance policies.

As can be seen from eq. (5) a Pareto-optimal risk allocation is achievable - under moral hazard - only if

a) $f_{a}(x, a) / f(x, a)=$ constant $=c$.

This implies, however, $\int f_{a}=\int f . c=c$, since $\int f=1$ for all $a$, hence $f_{a} \equiv 0$, contradicting the assumption that for $x_{a}<0=>f_{a}>0$, i.e. that the activity of the insured influences the outcomes (or losses) ; consequently perfect risk allocation could only obtain if

b) $\lambda=0$.

But, in fact, $\lambda>0$ since for $\mu>0$

$$
\mu \int(x-\Phi(x)) f_{a}(x, a) d x>0,
$$

3 To use the same utility function for the insurer and the insured is motivated by the hypothesis advanced in most insurance writings that " the company acts on behalf of the insured" (guaranteed e.g. by regulation laws). 
and the expression in waved brackets in eq. (7) is the second-order condition of the insured's maximization problem and is negative.

Now, eq. (5) shows that the deviation from the first-best optimum should be made in proportion to the ratio $f_{a}(x, a) f(x, a)$, whereby the risk aversion of the insured is taken into account, i.e. the deviation is the greater the more pronounced is the effect of the activity on the loss distribution. This, in turn, means that the insurer takes this ratio as a signal that the insured has not taken the right (or proper) activity.

This second-best solution results in incomplete coverage against loss, subjecting the insured to some financial risk. In other words, the deviations from the optimal risk sharing arrangements which seem to be necessary as incentives for the appropriate activities of the insured imply that he is made fully responsible for the outcomes though these are only partially within his activity space.

These departures from the first-best optimum point to the implicit costs of a contract under incomplete and asymmetric information. Therefore, there are to be expected positive gains in monitoring the activity of the insured, for in this case a 'forcing contract' (in the sense of Harris and Raviv [1978]) could be used to achieve a first-best optimum.

\section{Optimal contracts and the value of additional information}

Consider the following case : the insurer has no information about the activities of the insured, hence the coverage (or indemnity) depends on the outcome alone. Now, if the insured is risk-neutral the indemnity equals the loss minus a constant (the deductible). Such an indemnity function provides for the desired incentives of the insured, and allocates the risks in the best way (independently of the insurer's risk attitude). But if the insured is risk-averse then such an indemnity function burdens the insured with the risk of the outcome given his level of activity. If, instead, a fixed amount will be paid out to secure the insured against the risk, then there is no incentive to take certain actions. Consequently, in case the insureds are risk-averse the "optimal insurance policy" (i.e. the indemnity) depends always on the loss, but the insureds are not charged with the total risk (the variability of the outcome given his action). As Shavell [1979] has shown, the welfare loss of moral hazard is very likely small and the coverage almost complete if the costs of preventing losses either are very high or very small.

Consider now the second case: The insurer has information about the activity of the insured, such that the coverage (or indemnity) can depend on the outcome (i.e. the magnitude of the loss) as well as on the activity taken. In case the insured himself is risk-neutral nothing is lost if the indemnity depends only on the outcome. This is intuitively clear: Because the insureds are risk-neutral they act as perfect insurers against the risk of variability of the outcome minus the "costs" of the activity. Hence 
it is desirable for them to maximize this "net return"; a fixed deductible leads to this result owing to the insured's risk neutrality.

But if the insureds are risk-averse there is a real gain in making the indemnity partially dependent on the activity - for it is desirable to risk-averse actors to avoid risks as far as possible - provided the activity is perfectly monitored. In this case a first-best solution is possible by an insurance policy inducing (or enforcing) the "right activity (level) ".

In some simple cases perfect monitoring may be possible, and hence a first-best solution attainable with full coverage and optimal risk sharing (as long as the monitoring costs are negligible). In general, however, perfect monitoring of the insured's activity is impossible or prohibitively expensive. Optimal contracts depend then on the "technology of observation" indicating what is observable and to what costs.

However, if the activity is not accurately monitored then the use of incomplete information introduces a new risk, namely that the indemnity reflects an incorrect notion of the insured's true activity. As long as both the insured and the insurer are risk-averse this introduction of a new risk seems undesirable since this worsens welfare.

There is, therefore, a real problem to be solved: when is additional incomplete information useful ? Further, how can this information be used optimally?

There might exist a signal as an additional source of information to infer the activity of the insured. The question - as raised e.g. by Shavell [1979] is now : Does the new optimal insurance policy $\Phi(x, y)$ which uses this additional information strictly dominate (in the Pareto sense) the insurance policy $\Phi(x)$ depending only on the loss ?

This means, find a signal ( $y$ ) that could be observed besides the outcome (loss $x$ ) by both the insurer and the insured, and which could, therefore, be used in the construction of the insurance policy. The terms of the insurance policy are such that for the same value of the loss $(x)$ but different contingencies signalled by $y$, the coverage (or indemnity) of the insured should vary. This gives raise to the notion of value of the signal: A signal is valuable if both sides, the insured and the insurer could gain by using the signal in the terms of the insurance policy.

The signal, or monitor, is a random variable whose distribution is conditional on $a$. If the available signals have distributions which are independent of $a$, then it is clear that monitoring is not worthwhile, i.e. the signals are valueless. In other words, it is assumed that conditional on any outcome (loss), different levels of the insured's activity are associated with different probability density functions of the signal $f(y \mid x ; a)^{4}$.

\subsection{Inaccurate observation - Special monitors}

Recently these questions were analyzed by Harris and Raviv in two very stimulating papers [1978], [1979]. They conclude that the information is useful or valuable only under very restricted conditions. But then they are able to show "that all the results

4 If this were not true $f(y \mid x ; a)=f(y \mid x)$, and then $f(a \mid x ; y)=f(a \mid x)$. 
achievable under perfect information can be obtained even when information is imperfect, using a forcing contract" $(1978$, p. 25$)$. This type of contract specifies that the insured is indemnified only if the monitoring reveals his activity to be 'acceptable'. As a result, this is then equivalent to monitoring directly the insured's activity.

Harris and Raviv use the special monitor $z=a+\varepsilon$, that is, the insurer observes the activity $a$ plus a random variable $\varepsilon$ with zero mean and a positive density on an interval $[-b, c]$ for some finite $b, c$. In technical language this is called "noise". Harris and Raviv call such monitors regular monitors. If $z$ is any regular monitor, Harris and Raviv (1979, Proposition 7) show by adopting a proof of Shavell [1979] that the use of such a monitor allows realization of some of the gains of monitoring. They demonstrate further that it is always optimal to use the monitor in a dichotomous contract. That is, a contract of the form

$$
\Phi(x, z)=\left\{\begin{array}{l}
\Phi^{o}(x, z) \text { for } z \geqslant \hat{z} \\
w \text { otherwise, }
\end{array}\right.
$$

where $\left(\Phi^{o}, x, z\right)$ is a continous contract, $w$ is a real constant for which $U(w, a)$ is defined, and $\hat{z}$ is a real parameter. The insured is here indemnified according to some prespecified schedule (depending on the loss and the monitoring result) if his activity is judged to be acceptable, based on the monitoring result. Otherwise, if his activity level is judged to be unacceptable the insured receives a less preferred fixed payment, $w$; or in other words, the compensation may be refused and/or the policy cancelled. If the monitor is uniformly distributed on $[a-b, a+b]$ for some $b>0$, the optimal monitoring contract is of the form

$$
\Phi^{*}(x, z)=\left\{\begin{array}{l}
w \text { if } z \geqslant a^{*}-b \\
0 \text { otherwise }
\end{array}\right.
$$

where $w$ is a constant payment and $a^{*}-b$ is the minimum acceptable value of $z$. This implies, if $a+\varepsilon<a^{*}-b$ pay nothing, in all other cases the coverage is a fixed amount. As a result, the insured will choose $a=a^{*}$, the "right" or "appropriate" activity level.

As Shavell [1979] remarks, the case of the regular monitor holds under the general assumption that the probability distribution of $z$ is different for different $a$. He uses the cumulative probability distribution function of $z$ given $a, F(z ; a)$, and $f(z ; a)$, the density function of $z$ given $a$. Shavell then addresses to the further question "that observations are made, if at all, either ex ante, when a policy is purchased, or ex post, when a claim is presented" (p. 550). He proves that if the insurer monitors the activity with perfect accuracy then (a) "an optimal insurance policy under moral hazard involves full coverage", and (b) the activity " is observed ex post ... unless the relative cost of an ex ante observation is sufficiently small" (p. 553). If instead " the insurer's observations are made without cost and convey only imperfect information" about the insured's activity, he proves that (c) "either ex ante or ex post 
observations are of positive value - the terms of the insurance policy will depend to some extent on them", and (d) "ex ante observations are more valuable than ex post, at least when the quality of the two types of observations is the same" (p. 555).

\subsection{Inaccurate observation - General monitors}

But as argued above, the analysis of Harris and Raviv and Shavell depends essentially on there being a concave expected utility; this, however, is not very likely. Furthermore, the optimal solution has to be differentiable. Given the "observation technology" of Harris and Raviv we get, however, a non-differentiable ("corner") solution. In the following the theme is, therefore, pursued in the context of the second model, using the Mirrlees approach.

Now, suppose that $F(x, y ; a)$ is the joint distribution of $x$ and the signal $y$ given $a$; and $f(x, y ; a)$ is the density function ${ }^{5}$. As before it is assumed that $f, f_{a}$, and $f_{a a}$ exist. Then the following is an extension of eq. (5) in the case of a signal $y$

$$
\frac{\lambda U_{w a}-\mu}{U_{w}}=-\left[1+\lambda \frac{f_{a}(x, y ; a)}{f(x, y ; a)}\right] .
$$

If $f_{a}(x, y ; a) / f(x, y ; a)=h(x ; a)$ holds for all $a$, then - solving it as a differential equation in $a-$ yields

$$
f(x, y ; a)=g(x, y) \cdot h(x ; a) \quad \text { for all }(x, y) .
$$

Now, if (9) holds the signal is valueless since then $x$ contains all the relevant information about $a$. In other words, $x$ is a "sufficient statistic for the pair $(x, y)$ with respect to $a$ " (Holmström [1979], p. 84). That is, monitoring does not reveal further information about the activity level $a$ as already contained in $x$. But if (9) is false, then $y$ is a true signal (or monitor) and, therefore, should be used in the contract.

Normally, $f_{a}(x, y ; a) / f(x, y ; a)$ will change with $y$ implying that for a given $x$ the coverage for the insured should be different for different circumstances signalled by $y$. If in the extreme the influence of $y$ is such that for every $x, f_{a}(x, y ; a) \equiv 0$, then - as eq. (8) shows - this influence should be neglected, and the optimal (first-best) payment function applied, keeping constant the marginal utility of wealth under all contingencies or states.

It remains to proof the following proposition :

There exists an insurance policy $\Phi(x, y)$ which strictly Pareto dominates $\Phi(x)$ if and only if eq. (9) is false.

To proof this proposition (for details see the Appendix) take an additive variation $\delta \Phi(x, y)$ in the payment function $\Phi(x)$ such that the expected utility of the insured

5 A similar approach is followed by Holmström [1979]. For this reference I had to thank Dr. M. Dietsch (University of Lille). 
will be higher under the new contract $\Phi(x, y)$ than under $\Phi(x)$, and that the insurer will at least break-even under the new contract $\Phi(x, y)$.

Now, insofar as the signal is at all valuable - as indicated in eq. (9) - there "will be no first-order effect on the individual's expected utility that can be attributed to the imposition of risk because, initially, his coverage and thus his final wealth were fixed, conditional on there being a loss", as Shavell ([1979], p. 554) has formulated. That is, since the insured is "on the margin risk-neutral towards randomness in $y$, given $x$, the new contract can be designed so that marginally it does not increase risk" (Holmström [1979], p. 87). However, there will be a first-order effect on the activity taken by the insured lowering the probability of loss and therefore allowing a reduction in premiums. Consequently, insofar as the signal can be observed and embodied in the contract without costs, use any valuable signal regardless of how noisy it is.

To illustrate this concept of informativeness further, consider the following examples ${ }^{6}$.

First example : Suppose, $y$ is independent of $x$, that is the insured is directly observable, then

$$
f(x, y ; a)=g(y, a) \cdot h(x, a)
$$

and

$$
\frac{f_{a}(x, y ; a)}{f(x, y ; a)}=\frac{g_{a}(y, a)}{g(y, a)}+\frac{h_{a}(x, a)}{h(x, a)} .
$$

Hence, the signal $y$ is of no value if $g_{a} / g$ is constant, all relevant information is contained in the second part. This implies, however, that $g_{a} \equiv 0$ (for $\int g_{a}=0$ ). Hence, if $g$ depends at all on $a$, the signal $y$ is valuable.

Second example: If $y$ is valuable, then one can construct another "indicator" or signalling system with

$$
Y= \begin{cases}y \text { if } & |x| \geqslant \bar{x} \\ 0 \text { if } & |x|<\bar{x}\end{cases}
$$

that means, the new signal $Y$ is a "conditional indicator": Whenever the outcome is sufficiently high (i.e. the loss exceeds a certain amount $\bar{x}$ ) the signal $Y$ will be used and monitoring resources expended. If it is costly to monitor, then the signal $\boldsymbol{Y}$ will be used if it is cheaper than the initial signal $y$. This opens up also the possibility of a random indicator, an indicator used only at random. Casual observation of real contracts shows that the cost reduction is often sufficiently high to compensate for the information loss.

6 See also Holmström ([1979], p. 87). 


\section{Concluding remarks}

Moral hazard results from incomplete and asymmetrically distributed information : Insurance itself alters the incentives for specific actions (as, e.g. care), and therefore the probabilities of the outcomes. The outcomes are hence a mixture of action and (exogenous) uncertainty or risk.

Depending on what is observable the terms of the insurance policy change as the insurer wants to survive and consequently must respond. If the state of nature (exogenous risk) and/or the insured's activity is accurately observable or controllable, then there is no problem of moral hazard. This phenomenon also disappears if the insureds as agents are all risk-neutral. But if alone the outcome is observable, then optimal insurance policies are only of a second-best nature on account of moral hazard. The deviation from the first-best risk spreading is the greater the more pronounced is the effect of the action on the loss distribution. This implies that maluses and bonuses should be levied in proportion to this distance.

But these departures from the optimal risk sharing which seem to be necessary as incentives for the appropriate action (or behaviour) of the insured burden him unduly with excess responsibility for the outcome. By conceiving of signalling systems or indicators or using other available information about the insured's behaviour (e.g. the number of doctor visits or miles/year) or the (exogenous) risk, in general insurance policies can be improved and positive gains realized. A simple necessary and sufficient condition for being valuable is that the incomplete information or indicator carries over and above the information already contained in the losses or the loss distribution some further clues for the insured's (true) activity.

Additional information is valuable because the same incentives are produced with smaller deviations from the first-best risk allocation (and hence smaller losses from the inherent gains of risk sharing). This conclusion is reinforced by the fact that many contracts are long-term relationships. This intertemporal dimension permits the accumulation of evidence on the insured's behaviour, and, therefore, allowing to detect more easily divergent behaviour, and hence to mitigate the phenomenon of moral hazard.

\section{Appendix *}

To proof the if-part of the proposition, suppose $y$ is valueless. Now define for every $x$ a payment fuction $\Phi(x)$ which is at least as good as $\Phi(x, y)$ such that

$$
\begin{aligned}
\int U(\Phi(x, y), a) g(x, y) d y & =\int U(\Phi(x), a) g(x, y) d y \\
& =U(\Phi(x), a) \int g(x, y) d y
\end{aligned}
$$

Now, using eq. (9) and (A.1)

* Remark: This proof follows in the essentials the one given by Holmström [1979]. For valuable discussions I thank my colleague K.-H. Brodbeck. 


$$
\begin{aligned}
\int U(\Phi(x, y), a) f(x, y ; a) d x d y & =\int U(\Phi(x, y), a) h(x, a) g(x, y) d x d y \\
& =\int U(\Phi(x), a) h(x, a) g(x, y) d x d y
\end{aligned}
$$

That is, the insured will choose the same activity under $\Phi(x)$ as under $\Phi(x, y)$ since his utility is the same.

By Jensen's inequality, eq. (A.1) implies

$$
\int \Phi(x, y) g(x, y) d y \geqslant \int \Phi(x) g(x, y) d y
$$

or

$$
\int(x-\Phi(x, y)) g(x, y) d y \leqslant \int(x-\Phi(x)) g(x, y) d y .
$$

This is true for every $x$, and $h(x, a) \geqslant 0$, integration yields

$$
\int(x-\Phi(x, y)) f(x, y ; a) d x d y \leqslant \int(x-\Phi(x)) f(x, y ; a) d x d y
$$

The insured takes, by construction, the same activity under $\Phi(x)$ as under $\Phi(x, y)$, hence this shows, that the insurer is at least as well off with $\Phi(x)$ as with $\Phi(x, y)$. Thus $\Phi(x)$ is weakly Pareto superior to $\Phi(x, y)$ if eq. (9) is true.

To prove the only if part, fix $x$ for a moment, and choose a variation $\delta \Phi(x, y)$ of the second-best solution $\Phi(x)$ such that the marginal return for the insured $\left(\delta E^{i}\right)$ is positive and the insurance company $\left(\delta E^{c}\right)$ will at least break even, conditional on $x$.

Since the insured's response is unique these marginal returns are given by 1

$$
\delta E^{i}=U^{\prime}(\Phi(x), a) \int \delta \Phi(x, y) f(x, y ; a) d y
$$

and

$$
\begin{aligned}
\delta E^{c}= & U^{\prime}(\Phi(x), a) \int \delta \Phi(x, y) f(x, y ; a) d y-\mu \int \delta \Phi(x, y) f(x, y ; a) d y \\
& +\lambda\left[U^{\prime}(\Phi(x), a) \int \delta \Phi(x, y) f_{a}(x, y ; a) d y+U^{\prime}{ }_{a}(\Phi(x), a) \int \delta \Phi(x, y) f(x, y ; a) d y\right]
\end{aligned}
$$

Here $\lambda$ is the solution to eq. (7) corresponding to $\Phi(x)$. Now, if the signal $y$ is valuable, then it follows from eq. (9) that there is a set $Y$ in the range of $y$, with

$$
\int_{Y} f(x, y ; a) d y \equiv f(x, Y ; a) \neq 0 .
$$

Now, choose a variation $\delta \Phi(x, y)$ such that $\delta \Phi(x, y)>0$; here $\delta \Phi(x, Y)$ is constant for all $y \in Y$.

Under these conditions it follows that

$$
\delta E^{i}>0
$$

and

$$
\delta E^{c}=0
$$

1 See the Proposition 1, Section 9.6 in Luenberger [1969]; intuitively this proposition states that - in the first order - we can determine the variation in the functionals on a variation in the contract without evaluating the implicit function. 
if $\int \delta \Phi(x, y) f_{a}(x, y ; a) d y \neq 0$. From eq. (A.3) follows

$$
U^{\prime} \alpha-\mu \alpha+\lambda\left(U^{\prime}{ }_{a} \alpha+U^{\prime} \beta\right)=0
$$

or

$$
\alpha\left(U^{\prime}-\mu+\lambda U_{a}^{\prime}\right)=-\lambda U^{\prime} \beta
$$

if we take $\alpha=\int \delta \Phi(x, y) f(x, y ; a) d y$ and $\beta=\int \delta \Phi(x, y) f_{a}(x, y ; a) d y$. Since the expression in brackets is only zero if $f_{a}(x, a)=0$, which we have excluded in eq. (5), $\lambda, \mu$, and $U^{\prime} \neq 0$, as well as $\alpha$ and $\beta \neq 0$ ( $\beta=0$ was discussed on p. 29, with the result that the firstbest solution should be applied), we have proofed the necessity of our statement. To proof also the sufficiency suppose that $\int U(\Phi(x), a) f(x, a) d x$ is strictly convex. The procedure can be repeated for a set of $x$-values with positive mass, since $y$ is valuable. q.e.d.

\section{REFERENCES}

ARROW, K. J. [1970] : "Insurance, risk and resource allocation", in Essays in the Theory of Risk-Bearing, North-Holland, Amsterdam, 134-143.

EISEN, R. [1976] : "Unsicherheit und Information - Unkontrollierbares Verhalten und das Problem des moralischen Risikos", Jahrbücher für Nationalökonomie und Statistik, 191 (December 1976), 193-211.

EISEN, R. [1979] : Theorie des Versicherungsgleichgewichts - Unsicherheit und Versicherung in der Theorie des generellen ökonomischen Gleichgewichts, Duncker \& Humblot, Berlin.

HARRIS, M., and RAVIV, A. [1978] : "Some results on incentive contracts with application to education and employment, health insurance and law enforcement", American Economic Review, 68 (March 1978), 20-30.

HARRIS, M., and RAVIV, A. [1979] : "Optimal incentive contracts with imperfect information ", Journal of Economic Theory, 20 (April 1979), 231-259.

HOLMSTRÖM, B. [1979] : "Moral hazard and observability", The Bell Journal of Economics, 10 (Spring 1979), 74-91.

LUENBERGER, D. [1969] : Optimization by Vector Space Methods, Wiley, New York.

MIRRLEES, J. A. [1974] : "Notes on welfare economics, information and uncertainty", in Essays on Economic Behavior under Uncertainty, Balch, Mc Fadden and Wu (Eds.), North-Holland, Amsterdam, 243-261.

SHAVELL, S. [1979] : "On moral hazard and insurance", Quarterly Journal of Economics, 93 (November 1979), 541-562.

SPENCE, A. M., and ZECKHAUSER, R. [1971] : "Insurance, information, and individual action ", American Economic Review, 61 (May 1971), 380-387. 